\title{
Structural Analysis of Aileron Panel using Finite Element Method
}

\author{
Sriram Bharath ${ }^{1}$, N.V.Swamy Naidu ${ }^{2}$ \\ ${ }^{I}$ (PhD Scholar Department of Mechanical Engineering, GITAM University, Hyderabad, India.) \\ ${ }_{2}^{2}$ (Professor-HOD Department of Aerospace Engineering, GITAM University, Hyderabad, India)
}

\begin{abstract}
Analysis of Composites subjected to different loading conditions is the subject of research interest in recent years. Various works have been published on analysis of Composites in the recent years. Due to the orthotropic structural behavior of Composites, the prediction of structural response is a challenging problem in the analysis of laminated composites. Several studies have been done on structural analysis of Composite materials using four node elements. A study is made on the structural analysis of Aileron Panel made up of composite material subjected to uniform pressure load using Finite element Method. In this paper, Aileron Panel is assumed as a rectangular plate type structure made of composite material. An Eight-node isoparametric quadratic element is used for the analysis. MATLAB code has been developed to find the deflection of Aileron Panel. A parametric study is carried out varying the thickness of Aileron with simply supported and clamped support conditions.
\end{abstract}

Keywords - Composite, Finite Element Analysis, Eight Node element, MATLAB.

\section{Introduction}

An Aileron is part of the trailing edge of each wing of a fixed-wing aircraft which is a hinged flight control surface. Ailerons are used in pairs to control the aircraft in roll (or movement around the aircraft's longitudinal axis). Aileron is usually made of composites. In this paper it is assumed that Aileron having 10 ribs and it is unbuckled section of panel in $0.75 \mathrm{mX} 1.592 \mathrm{~m}$ rectangular plate type structure.

Composites suit any function because of their extremely versatility. As Composites are light weight with high specific strength \& high specific Stiffness along with corrosion resistant Composites are extensively used from aerospace industry to common everyday applications.

A number of plate theories have been developed to predict the structural behavior of composite plates. Transverse shear stresses are neglected In the classical laminate plate theory (CLPT), therefore the plane sections normal to the mid surface remain same before and after deformation.

A review of various equivalent single layer and layer wise laminated plate theories was presented by Reddy and Robbins [9]. An overall comparison of laminated theories based on displacement hypothesis was presented by Liu and Li [10], including shear deformation theories, layer wise theories, generalized Zigzag theories, and the proposed global-local double-superposition theories. A review of theories for laminated and a sandwich plate was presented by Altenbach [12]. A review of displacement and stress-based refined shear deformation theories of isotropic and anisotropic laminated plate was given by Ghugal and Shimpi [13].

\section{Formulation}

A composite laminate is considered consisting of a thin homogeneous and orthotropic/isotropic layers oriented arbitrarily and having a total thickness of $\mathrm{h}(\mathrm{h} 1, \mathrm{~h} 2, \mathrm{~h} 3$ etc. are thicknesses of individual layers.

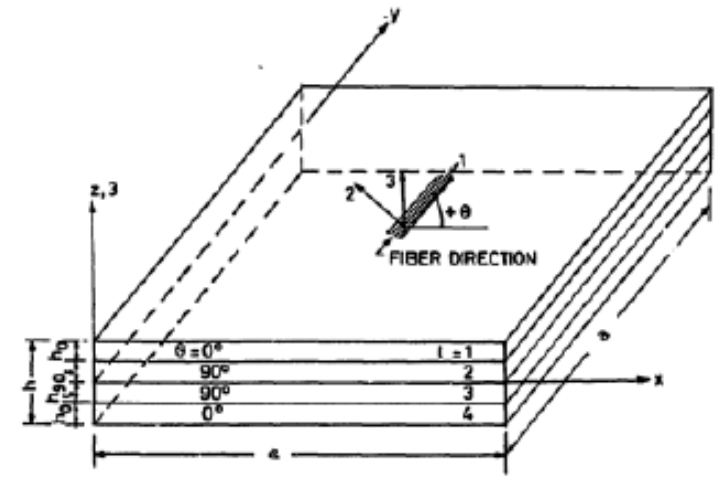

Fig.1 Geometry of a four-layer symmetric laminate. 
The $\mathrm{x}$-y plane coincides with the middle plane of the laminate with the $\mathrm{z}$-axis oriented in the thickness direction such that $\mathrm{x}, \mathrm{y}$ and $\mathrm{z}$ form a right-handed screw coordinate system. In the present theory, the displacement components of a generic point in the laminate are assumed to be of the form (see Kant and Pandya [7] and Reddy [5] )

Where displacements are

$$
\begin{aligned}
& u(x, y, z)=u_{0}(x, y)+z \theta_{x}(x, y) \\
& v(x, y, z)=v_{0}(x, y)+z \theta_{y}(x, y) \\
& w(x, y, z)=w_{0}(x, y)
\end{aligned}
$$

The terms $\mathrm{u}_{0}, \mathrm{v}_{0}$ and $\mathrm{w}_{0}$ are the mid-surface displacements. They are not the same as the neutral bending axis displacements presented in strength of materials discussions for beams made of isotropic materials and the strain terms are given below

$$
\begin{aligned}
& \varepsilon_{x}=\frac{\partial u_{0}}{\partial x}+z \frac{\partial \phi}{\partial x} \\
& \varepsilon_{y}=\frac{\partial v_{0}}{\partial x}+z \frac{\partial \psi}{\partial x} \\
& \varepsilon_{z}=\frac{\partial w}{\partial z} \\
& \gamma_{x z}=\frac{\partial w_{0}}{\partial x}+\phi \\
& \gamma_{y z}=\frac{\partial w_{0}}{\partial y}+\psi \\
& \gamma_{x y}=\left(\frac{\partial v_{0}}{\partial x}+\frac{\partial u_{0}}{\partial y}\right)+z\left(\frac{\partial \phi}{\partial y}+\frac{\partial \psi}{\partial x}\right)
\end{aligned}
$$

The nonzero mid-surface strains are defined as

$$
\left\{\varepsilon^{0}\right\}=\left\{\begin{array}{c}
\varepsilon_{x}{ }^{0} \\
\varepsilon_{y}{ }^{0} \\
\gamma_{x y}{ }^{0}
\end{array}\right\}=\left\{\begin{array}{c}
\partial u / \partial x \\
\partial v / \partial y \\
\partial u / \partial y+\partial v / \partial x
\end{array}\right\}
$$

The mid-surface may experience curvatures related to the radius of curvature of the mid-surface. The curvatures are related defined as

$$
\left\{\begin{array}{l}
k_{x} \\
k_{x} \\
k_{x y}
\end{array}\right\}=\left\{\begin{array}{c}
\partial \phi / \partial x \\
\partial \psi / \partial y \\
\partial \varphi / \partial y+\partial \varphi / \partial x
\end{array}\right\}
$$

The strain variation through a laminate is expressed by a combination of equations (2) and (3) as 


$$
\left\{\begin{array}{c}
\varepsilon_{x} \\
\varepsilon_{y} \\
\gamma_{x y}
\end{array}\right\}=\left\{\begin{array}{c}
\varepsilon_{x}{ }^{0} \\
\varepsilon_{y}{ }^{0} \\
\gamma_{x y}{ }^{0}
\end{array}\right\}+z\left\{\begin{array}{l}
k_{x} \\
k_{x} \\
k_{x y}
\end{array}\right\}
$$

Where

$$
\phi=-\frac{\partial w_{0}}{\partial x} \quad \& \quad \psi=-\frac{\partial w_{0}}{\partial x}
$$

It follows directly from equation (3) that the curvatures are

$$
\left\{\begin{array}{l}
k_{x} \\
k_{x} \\
k_{x y}
\end{array}\right\}=\left\{\begin{array}{c}
-\partial^{2} w_{0} / \partial x^{2} \\
-\partial^{2} w_{0} / \partial y^{2} \\
-2^{\partial^{2} w_{0}} / \partial x \partial y
\end{array}\right\}
$$

The strain variation through a laminate is a function of both mid-surface strain and curvature and is continuous through the plate thickness. The stress need not be continuous through the plate. Consider the plane stress relationship between Cartesian stresses and strains.

$$
\left.\left\{\begin{array}{l}
\sigma_{x} \\
\sigma_{x} \\
\tau_{x y}
\end{array}\right\}=[\bar{Q}]\right\}\left\{\begin{array}{c}
\varepsilon_{x} \\
\varepsilon_{y} \\
\gamma_{x y}
\end{array}\right\}
$$

In order to establish the state of stress at a point in a laminate, the state of strain at the point must first be defined. Combining this state of strain with an appropriate constitutive relation yields the stress. For general loading conditions it is convenient to work with Cartesian components of stress and strain. For a specific lamina (termed the " $\mathrm{k}$ " lamina) the appropriate constitutive relationship is $[Q] \mathrm{k}$. Under conditions of plane stress the Cartesian components of stress in the $\mathrm{k}^{\text {th }}$ layer are

$$
\left\{\begin{array}{l}
\sigma_{x} \\
\sigma_{y} \\
\tau_{x y}
\end{array}\right\}_{k}=[\bar{Q}]\left(\left\{\begin{array}{c}
\varepsilon_{x} \\
\varepsilon_{y} \\
\gamma_{x y}
\end{array}\right\}+z\left\{\begin{array}{l}
k_{x} \\
k_{x} \\
k_{x y}
\end{array}\right\}\right)
$$

To satisfy conditions of equilibrium, resultant laminate forces must be balanced by the integral of stresses over the laminate thickness. The balance of forces (assuming $\sigma \mathrm{Z}=0$ ) is

$$
\left\{\begin{array}{l}
N_{x} \\
N_{x} \\
N_{x y} \\
Q_{x} \\
Q_{y}
\end{array}\right\}=\int_{-h / 2}^{h / 2}\left\{\begin{array}{l}
\sigma_{x} \\
\sigma_{y} \\
\tau_{x y} \\
\tau_{x z} \\
\tau_{y z}
\end{array}\right\} d z
$$


Moments are related to forces through a simple relationship of force time's distance. The balance of resultant moments yields

$$
\left\{\begin{array}{l}
M_{x} \\
M_{x} \\
M_{x y}
\end{array}\right\}=\int_{-h / 2}^{h / 2} z\left\{\begin{array}{l}
\sigma_{x} \\
\sigma_{x} \\
\tau_{x y}
\end{array}\right\} d z
$$

Equations (8) and (9) can be expressed in terms of the stresses in each layer. Since the kth layer is assumed to occupy the region between $\mathrm{Zk}$ and $\mathrm{Zk}-1$, it follows directly that equations (8) and (9) can be expressed as

$$
\begin{aligned}
& \left\{\begin{array}{l}
N_{x} \\
N_{y} \\
N_{x y}
\end{array}\right\}=\sum_{k=1}^{N} \int_{Z_{k-1}}^{Z k}\left\{\begin{array}{l}
\sigma_{x} \\
\sigma_{y} \\
\tau_{x y}
\end{array}\right\}_{k} d z \\
& \left\{\begin{array}{l}
Q_{x} \\
Q_{y}
\end{array}\right\}=\sum_{k=1}^{N} \int_{Z_{k-1}}^{Z k}\left\{\begin{array}{l}
\tau_{x z} \\
\tau_{y z}
\end{array}\right\} d z \\
& \left\{\begin{array}{l}
M_{x} \\
M_{x} \\
M_{x y}
\end{array}\right\}=\sum_{k=1}^{N} \int_{Z_{k-1}}^{Z k} z\left\{\begin{array}{l}
\sigma_{x} \\
\sigma_{y} \\
\tau_{x y}
\end{array}\right\} d z
\end{aligned}
$$

Where $\mathrm{N}$ is the total number of lamina in the laminate. The $Q_{x}$ and $Q_{y}$ have been segregated from the other load terms, for reasons to be subsequently discussed. Substituting equation (7) into the preceding equations, the laminate loads and moments are expressed in terms of the mid-surface strains and curvatures as

$$
\begin{aligned}
& \left\{\begin{array}{l}
N_{x} \\
N_{y} \\
N_{x y}
\end{array}\right\}=\sum_{k=1}^{N}[\bar{Q}]_{k}\left(\int_{z_{k-1}}^{z k}\left\{\begin{array}{c}
\varepsilon_{x}^{0} \\
\varepsilon_{y}^{0} \\
\gamma_{x y}^{0}
\end{array}\right\} d z+\int_{z_{k-1} z}^{z k}\left\{\begin{array}{l}
k_{x} \\
k_{x} \\
k_{x y}
\end{array}\right\} d z\right) \\
& \left\{\begin{array}{l}
M_{x} \\
M_{y} \\
M_{x y}
\end{array}\right\}=\sum_{k=1}^{N}[\bar{Q}]_{k}\left(\int_{z_{k-1}}^{z k}\left\{\begin{array}{l}
\varepsilon_{x}^{0} \\
\varepsilon_{y}^{0} \\
\gamma_{x y}^{0}
\end{array}\right\} d z+\int_{z_{k-1}}^{z k} z^{2}\left\{\begin{array}{l}
k_{x} \\
k_{x} \\
k_{x y}
\end{array}\right\} d z\right)
\end{aligned}
$$

Since the mid-surface strains $\{\varepsilon 0\}$ and curvatures $\{k\}$ are independent of the $\mathrm{z}$ coordinate, the integration is simplified. The integrals in equations (13) and (14) become simple integrals of (1, z, z2). The loads and moments can be expressed in matrix form. after integration, as Each

$$
\left\{\begin{array}{l}
N_{x} \\
N_{y} \\
N_{x y} \\
M_{x} \\
M_{y} \\
M_{x y}
\end{array}\right\}=\left[\begin{array}{llllll}
A_{11} & A_{12} & A_{16} & B_{11} & B_{12} & B_{16} \\
A_{12} & A_{22} & A_{26} & B_{12} & B_{22} & B_{26} \\
A_{16} & A_{26} & A_{66} & B_{16} & B_{26} & B_{66} \\
B_{11} & B_{12} & B_{16} & D_{11} & D_{12} & D_{16} \\
B_{12} & B_{26} & B_{26} & D_{12} & D_{22} & D_{26} \\
B_{16} & B_{26} & B_{66} & D_{16} & D_{26} & D_{66}
\end{array}\right]\left\{\begin{array}{l}
\varepsilon_{x}^{0} \\
\varepsilon_{y}^{0} \\
\gamma_{x y}^{0} \\
k_{x} \\
k_{y} \\
k_{x y}
\end{array}\right\}
$$


Each component of the $[A],[B]$, and $[D]$ matrices is defined by

$$
\begin{aligned}
& {\left[A_{i j}\right]=\sum_{k=1}^{n}\left[\bar{Q}_{i j}\right]_{k}\left(z_{k}-z_{k-1}\right)} \\
& {\left[B_{i j}\right]=\frac{1}{2} \sum_{k=1}^{n}\left[\bar{Q}_{i j}\right]_{k}\left(z_{k}^{2}-z_{k-1}^{2}\right)} \\
& {\left[D_{i j}\right]=\frac{1}{3} \sum_{k=1}^{n}\left[\bar{Q}_{i j}\right]_{k}\left(z_{k}^{3}-z_{k-1}^{3}\right)}
\end{aligned}
$$

The subscripts $i$ and $j$ are matrix notation, not tensor notation. The form of equation (15) is often simplified to

$$
\left\{\begin{array}{l}
N \\
M
\end{array}\right\}=\left\{\begin{array}{ll}
A & B \\
B & D
\end{array}\right\}\left\{\begin{array}{l}
\varepsilon^{0} \\
k
\end{array}\right\}
$$

When using this abbreviated form of the laminate load-strain relationship, where $(\mathrm{N}),(\mathrm{M}),(\varepsilon 0)$, and $(k)$ are offaxis quantities. They define laminate behavior with respect to the Cartesian (x-y) coordinate system. Each of the $3 \times 3$ matrices in either equation (15) or (17) has a distinct function identified by examination of equation (17). These matrices are termed:

$\left[A_{i j}\right]=$ extensional stiffness matrix

$\left[B_{i j}\right]=$ extension-bending coupling matrix

$\left[D_{i j}\right]=$ bending stiffness matrix

The expression for $Q_{x}$ and $Q_{y}$, can be written in a manner analogous to equations (15) or (17)

$$
\left\{\begin{array}{l}
Q_{x} \\
Q_{y}
\end{array}\right\}=\left[\begin{array}{ll}
A_{55} & A_{45} \\
A_{45} & A_{44}
\end{array}\right]_{k}\left\{\begin{array}{l}
\gamma_{x z} \\
\gamma_{y z}
\end{array}\right\}_{k}
$$

Following the same procedures as before, it can be shown that

$$
\left[A_{i j}\right]=C \sum_{k=1}^{n}\left[\bar{Q}_{i j}\right]_{k}\left\{\left(z_{k}-z_{k-1}\right)-\frac{4}{3 h^{2}}\left(z_{k}^{3}-z_{k-1}^{3}\right)\right\}
$$

Where $\mathrm{i}, \mathrm{j}=4,5$ and $\mathrm{h}$ is the total laminate thickness.

\section{Discussion and Conclusion}

In this paper dimensions are taken from Ref[16], Aerofoil NACA 64-210; Span-60m;Sweep angle-30 Deg; Aspect ratio-8.7;Sweep at c/4 point0-25.51 deg; Taper ratio-0.4;Root chord-10m;Tip chord-4m;Max. thickness of airfoil- $10 \%$ of chord; Aileron location- $6 \mathrm{~m}$ from tip; Flap location- $8.7 \mathrm{~m}$ from root; Mean aerodynamic chord$7.43 \mathrm{~m}$; Aileron span-7.5m; Flap span-3.3mWing. 


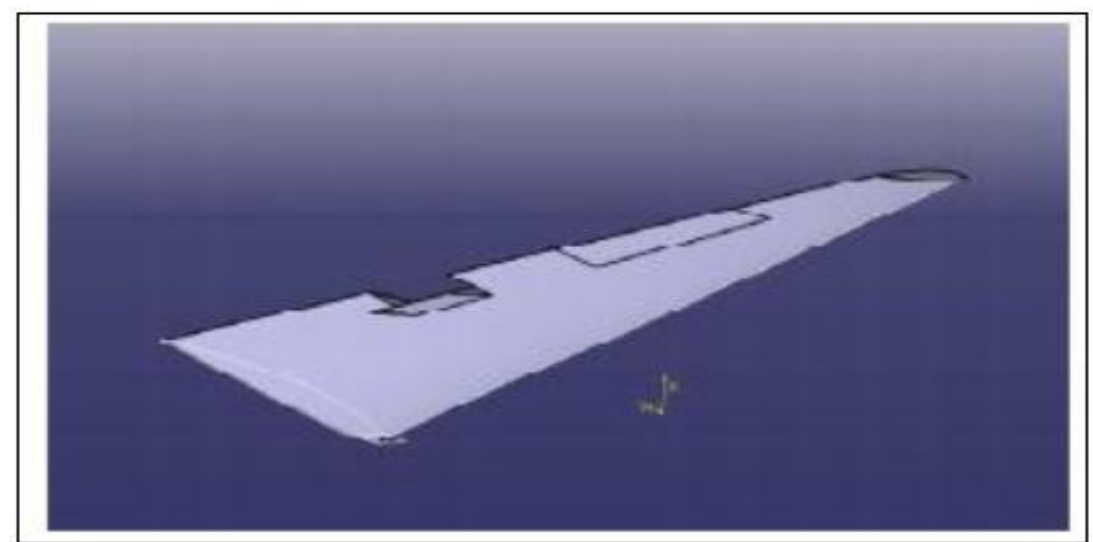

Fig.2 wing model with ribs and spars, twisted wing assembly

The deflection of Aileron panel is studied for Q8 isoparametric quadratic element, here Aileron panel is assumed to be made of composite material with 4 layers of different orientations $(0,45,90)$. Material properties of Graphite Epoxy are considered as E1/E2=25 G12=5 G13=5 G23=2 subjected to uniform pressure load of $5.446 \mathrm{~N} / \mathrm{m} 2$, the deflection of Aileron panel are listed in table for different $\mathrm{a} / \mathrm{h}$ ratio with different mesh sizes.

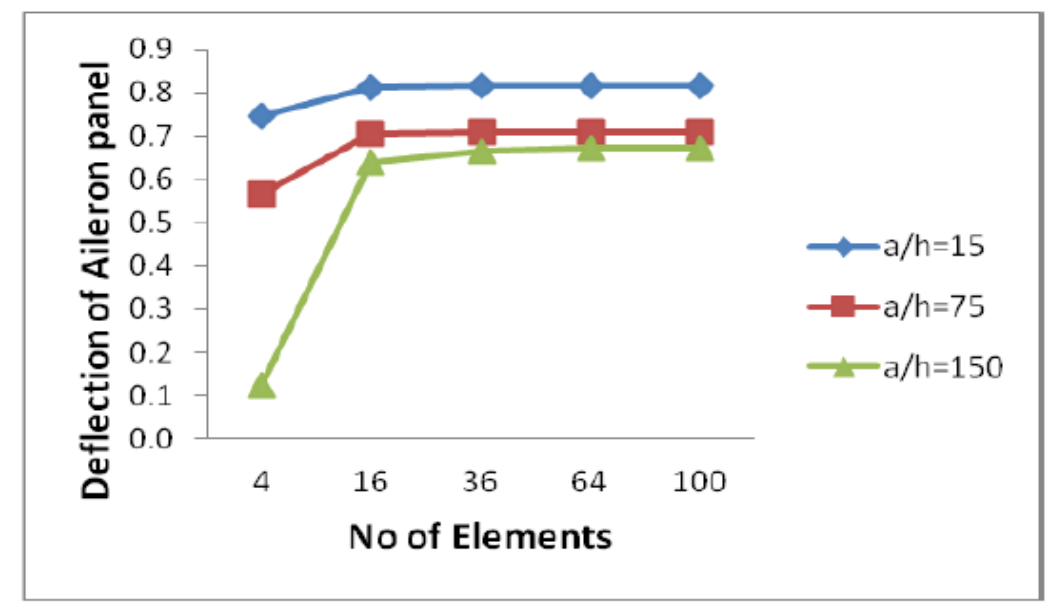

Fig.3 Deflection of Aileron panel with SSSS (0/90/90/0)s

From the above results it is clear that convergence is obtained at $8 \mathrm{X} 8 \mathrm{mesh}$, further parametric study is done for $8 \mathrm{X} 8$ mesh for various composite layer stacking sequences

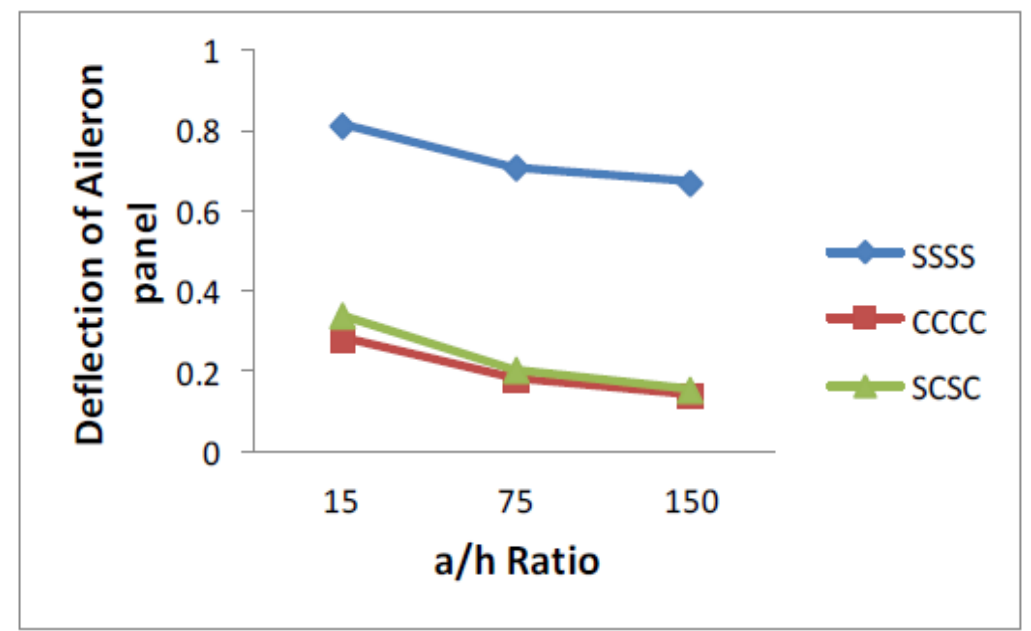

Fig.4 Deflection of Aileron panel (0/90/90/0)s 


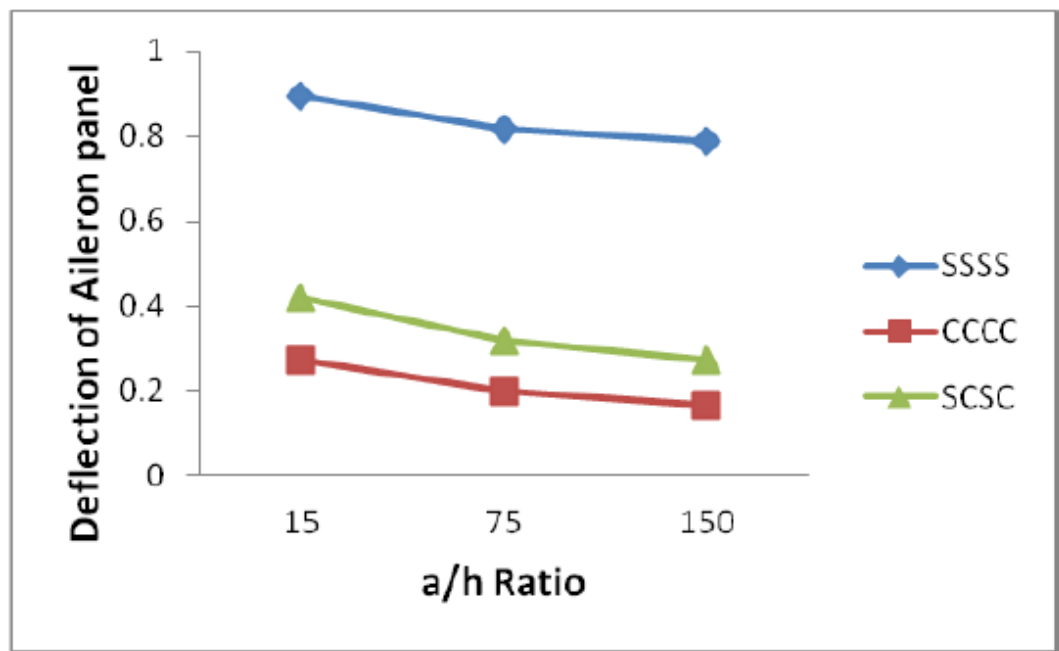

Fig.5 Deflection of Aileron panel (0/90/0/90)s

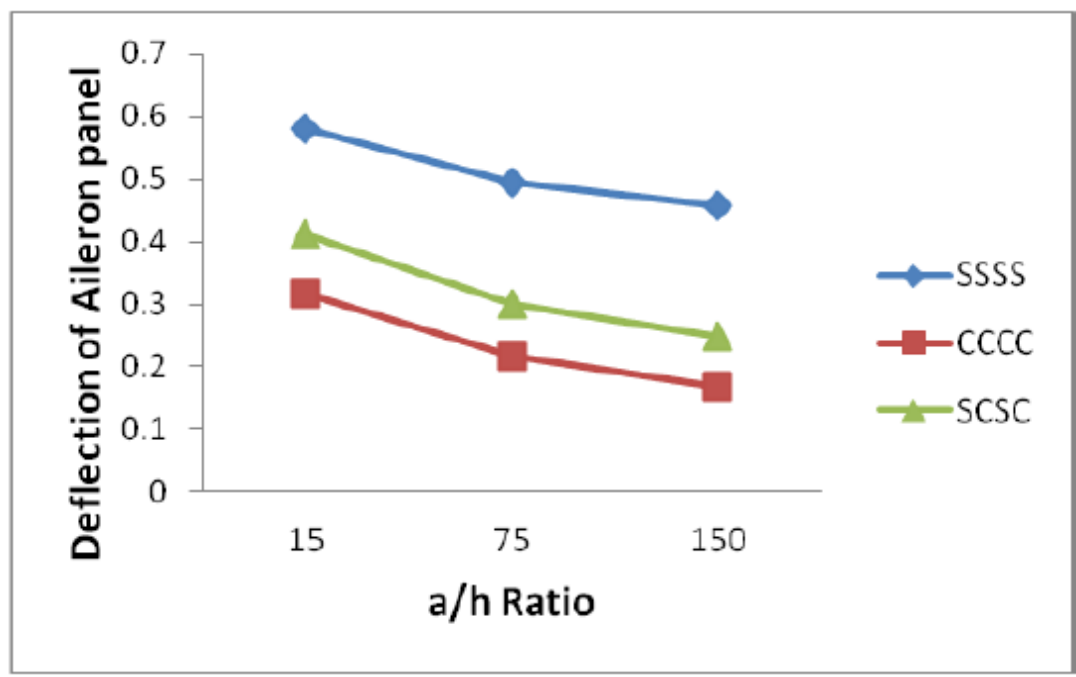

Fig.6 Deflection of Aileron panel (45/-45/-45/45)s

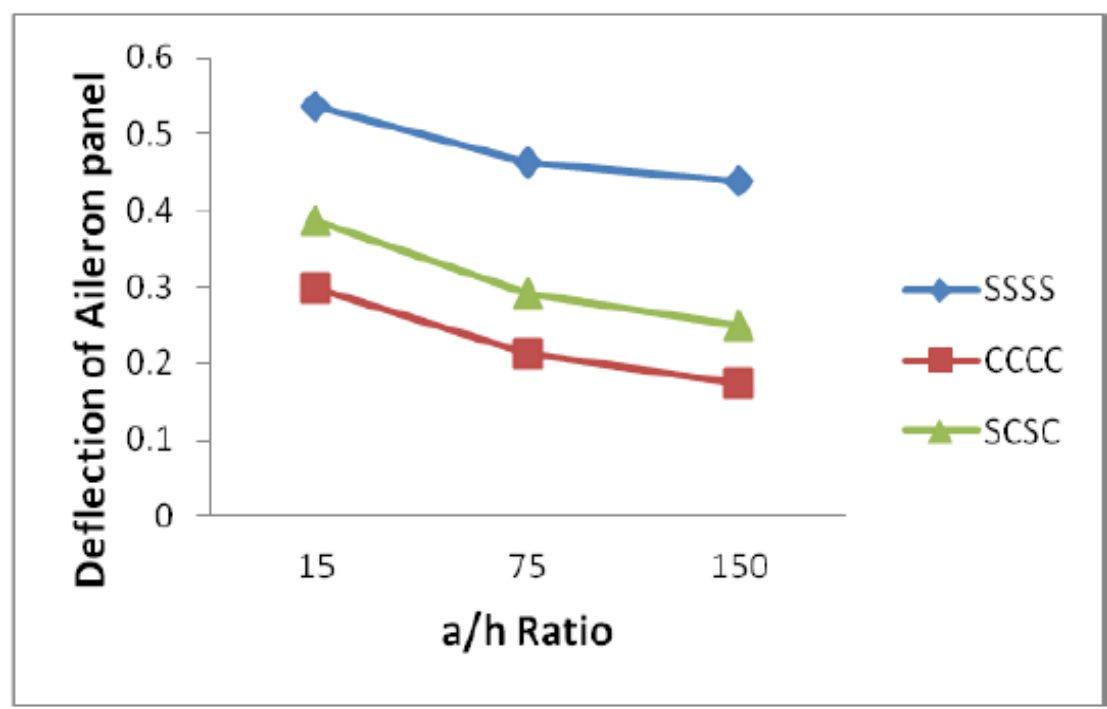

Fig.7 Deflection of Aileron panel (45/-45/45/-45)s 


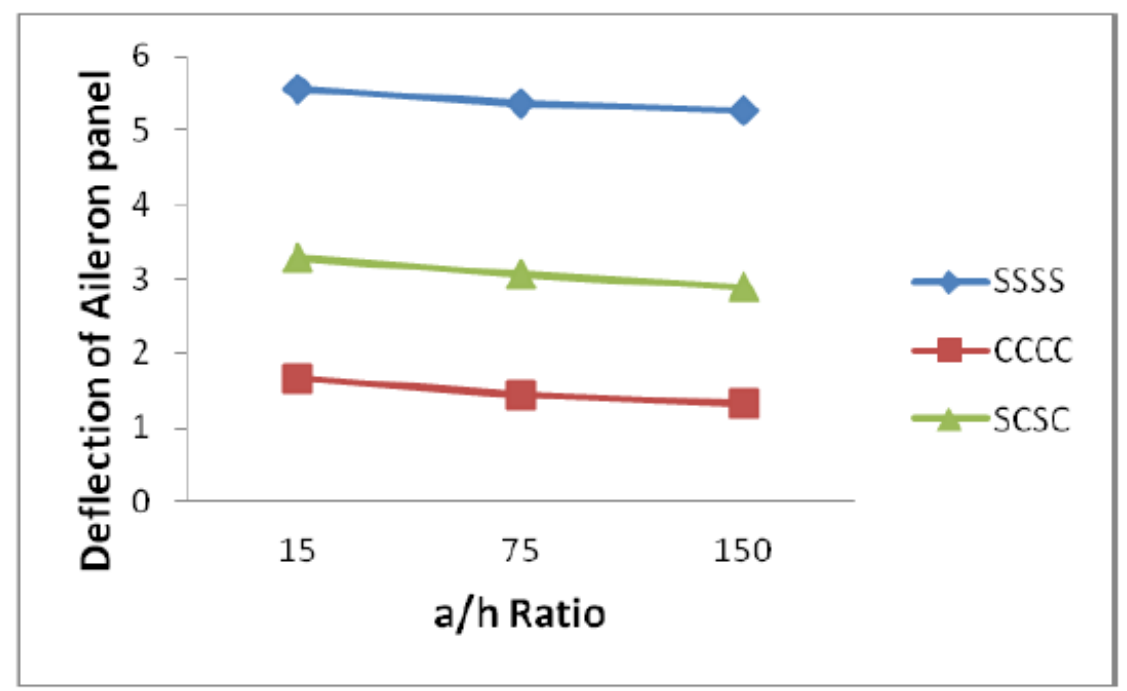

Fig.8 Deflection of Aileron panel (45/-45/0/90/90/0/-45/45)s

An Aileron panel is analyzed based on the uniform load applied, different boundary condition and side to thickness ratios. MATLAB is used for computing solution and get results regarding the deformation of Aileron panel. Figure 4 to 8 that the central deflection decreases with increase of a/h ratios of all different laminate stacking sequences and boundary condition.

\section{References}

[1]. Ambartsumyan SA. A new refined theory of anisotropic shells. Inst Math Mech, Acad Sci Arm SSR, Ere Trans Mekh Poli 1970;5:884-96.

[2]. Asghari, M., Kahrobaiyan, M.H., Ahmadian, M.T., 2010. A nonlinear Timoshenko beam formulation based on the modified couple stress theory. Int. J. Eng. Sci. 48, 1749-1761.

[3]. E. Reissner, "The Effect of Transverse Shear Deformation on the Bending of Elastic Plates", J. Appl. Mech., 12(1945),pp. 69-77.

[4]. R. D. Mindlin, "Influence of Rotatory Inertia and Shear on Flexural Motions of Isotropic, Elastic Plates", J. Appl.Mech., 18(1951), pp. 31-38.

[5]. J. N. Reddy, “A Simple Higher Order Theory for Laminated Composite Plates”, J. Appl. Mech., 51(1984), pp. 745752.

[6]. J. N. Reddy, A simple higher order theory for laminated composite plates. ASME J. dppl. Mech. 51, 745-752 (1984).

[7]. T. Kant and B. N. Pandya, A simple finite element formulation of a higher-order theory for unsymmetrically laminated composite mates. Compos. Strut. 9. 215-246 (1988).

[8]. Noor AK, Burton WS. Assessment of computational models for multilayered anisotropic plates. Composite Structure 1990;14:233-65.Xia,

[9]. Reddy JN, Robbins Jr DH. Theories and computational models for composite laminates Appl Mech Rev 1994;47:147-69.

[10]. Liu DS, Li XY. An overall view of laminate theories based on displacement hypothesis. J Compos Mater 1996;30:1539-61.

[11]. Noor AK, Burton WS, Bert CW. Computational models for sandwich panels and shells. Applied Mechanics Review 1996;49:155-99.

[12]. Altenbach H. Theories for laminated and sandwich plates, a review.Mech Compos Mater 1998;34(3):, $243-152$.

[13]. Ghugal YM, Shimpi RP. A review of refined shear deformationtheories of isotropic and anisotropic laminated plates. J Reinf Plast Compos 2001;20:255-72.

[14]. Prof.P.C.Pandey, Dept. of Civil Eng. (2004), composite materials [Web-based Course],

[15]. Xia., W., Wang, L., Yin, L., 2010. Nonlinear non-classical microscale beams: static bending, postbuckling and free vibration. Int. J. Eng. Sci. 48, 2044-2053.

[16]. N. Rajamurugu, S. Yaknesh, J. Anbarasi3 "Influence of Control Surfaces in Aircraft Wing Control Reversal Problems-FEA and CFD Analysis ". 